\title{
The future medicine of childhood obesity
}

\begin{abstract}
Children obesity has become one of the most important public health problems in many countries worldwide with some special concern towards industrial countries e.g.; USA and Western Europe, recently we came across many researches dealing with children obesity both in clinical and basic research fields explore the fact of childhood obesity related-comorbidities such as Non- alcoholic fatty liver disease (NAFLD) steatohepatitis (NASH) and early heart diseases, furthermore considering bariatric surgical intervention should be considered in morbidly -obese children still a matter of debate. Recently vertically transmitted microbes may initiate the obesity/ anti-obesity process early childhood, however in this communication I would like to put forth a critical clinical feedback of extremely hot point of ongoing clinical and academic researches. Hopefully will help everybody involved in the field.
\end{abstract}

Keywords: children obesity, vertical transmission, Elbanna, bariatric surgery, weight loss
Volume 5 Issue 4 - 2016

\author{
Abduh Elbanna,' Ali Ismael, ${ }^{2}$ Mohammad M \\ Sallam, ${ }^{2}$ Abd Elrazek ${ }^{3}$ \\ 'Department of General, laparoscopic and Obesity Surgery,Al \\ Azhar University, Egypt \\ ${ }^{2}$ Department of Internal Medicine, Zagazig University, Egypt \\ ${ }^{3}$ Department of Gastroenterology and Tropical Medicine,Aswan \\ University, Egypt
}

Correspondence: Abd Elrazek Mohammad Ali Hussein, Department of GIT,Tropical and Hepatology, Aswan School of Medicine, Aswan University, Aswan, Egypt PO 8I528, Egypt, Email ahmadrazek@gmail.com

Received: November 18, 2016 | Published: December 09, 2016

\section{Discussion}

Although, the awareness of childhood obesity as a modifiable health risk is high, many societies do not prioritize this issue as a health care problem, which may lead to comorbidities and even pre-mature death. Being overweight was also associated with decreased survival in several studies. Unfortunately, obese subjects are often exposed to public disapproval because of their fatness which significantly affects their psychosocial behavior. According to the World Health Organization (WHO), there are about 500million obese adults and 42 million obese children under the age of five worldwide. In the United States alone, 35 percent of adults (roughly 120 million people) and 17 percent of children are obese. Children obesity may begin from intrauterine life and through birth canal delivery. Childhood obesity is the mainstay problem today and its complications should be considered in early future, accordingly the world is going to establish medical and surgical solutions may prevent diseases related-obesity. Having one obese parent increases the risk of obesity by two- to threefold, and up to 15 -fold if both parents is obese. Obesity is also more prevalent among low-income populations. As an example in USA, (14.9) percent of low-income preschool-aged children were obese in 2010, as compared with (12.1) percent in this age group in the general population. ${ }^{1-8}$ High dietary calories, fatty meals and sedentary life increased prevalence of childhood obesity worldwide.

Additionally dietary inclusion of Lactobacillus, which belongs to Bacteroidetes and/or Bifidobacterium, can improve obesity both in murine model and humans, accordingly the intentional manipulation of community structure of gut microbiota may be a novel strategy to treat obesity. Bifidobacterium is one of the most numerous "probiotic" in mammalian gut among commensal bacteria, which belongs to Actinomycetes and also a kind of lactic acid bacteria. It can help Bacteroides degrade polysaccharides in a way that prevent obesity. However Bifidobacteria represent the dominant members of the gut microbiota of infants, despite the finding that they have been found to persist at high levels in some adult individuals, recently Milani and colleagues were able to trace the occurrence of a specific strain being present in the gut of both mother and infant. The hypothesis the obesity-anti obesity related microbiota should be considered a rout of transmission, ${ }^{9-11}$ hence the same Gut microbiota founded in both infant and related mothers will shed light on a new concept of mother to infant obesity and Anti-obesity transmission, furthermore, the microbiota translocations is the mainstay of metabolic syndrome in many obese patients. ${ }^{12,13}$ Additionally, it will be helpful if we know the BMI of all women included in Milani study, ${ }^{1}$ and if there is a correlation of Gut Bifidobacteria in both mother and child and if following the children to discover related- childhood obesity in suspected individuals. Interestingly, human milk is also associated with neonatal intestinal colonization by the beneficial microbes of the Bifidobacteria and Lactobacillus species rather than potential enteropathogenic bacteria such as Streptococci and Escherichia coli. Bifidobacteria and Lactobacillus species are the usual microbial agents used in probiotic preparations, which have been used to prevent necrotizing enterocolitis, and to treat colic and gastroenteritis in children. ${ }^{14,15}$ Such knowledge encouraging breast feeding may help prevent obesity, obesity associated-morbidities and infectious disorders during childhood.

Our preliminary/theoretical data suggested the good balancedrelation between fermicute and bacteriotete will maintain body weight index after some bariatric surgery (Not yet published data) hence some bariatric procedures would be concerned rather than other surgeries, not to regain weight after long term surgery (Figure 1).

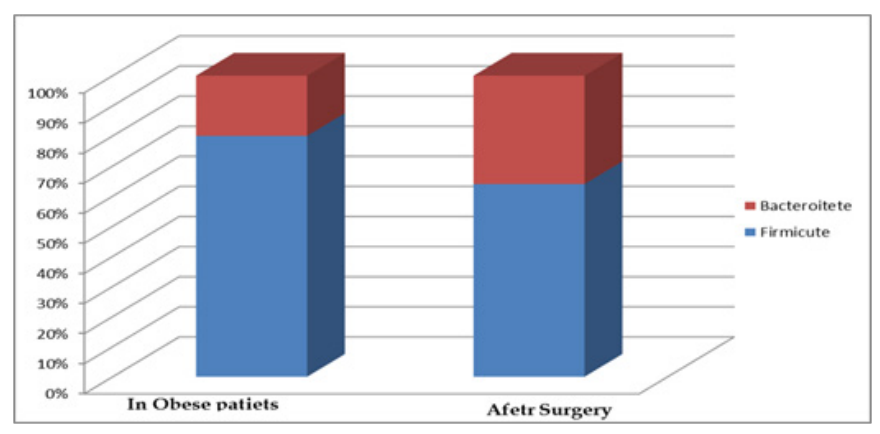

Figure I Balance of Bacteroitede and Fermicute during obesity and after surgery. 
However, bariatric surgery to treat childhood obesity is passing through a plateau phase, and the medical management and followup of children who have undergone bariatric surgery is a challenge. Unfortunately, there are no reports on the overall success rates or complications following bariatric bypass surgery in childhood obesity due to a lack of data. With the increased use of bariatric surgery to treat obesity in children, some guidelines have been published, most of which exclude children younger than 14years. Only few reports describe laparoscopic Sleeve Gastrectomy (LSG) in children and adolescents, nevertheless its safety and effectiveness have not been clarified yet, additionally no guidelines have been developed. Recently A novel bariatric surgical technique published in Medicine $($, Baltimore, USA, by Elbanna and his colleagues showed the first ever bariatric bypass to treat complicated childhood obesity. ${ }^{16-20}$ Elbanna operation shows promise in adulthood bariatrics. This technique is designed to maintain good digestion, better satiety and selective absorption with fewer medical and surgical complications. The Elbanna procedure preserves the duodenum, proximal jejunum, and terminal Ileum, along with the anatomical biliary drainage and enterohepatic circulation. In addition, fundal resection is performed to obtain the maximum weight loss; (Figure 2).

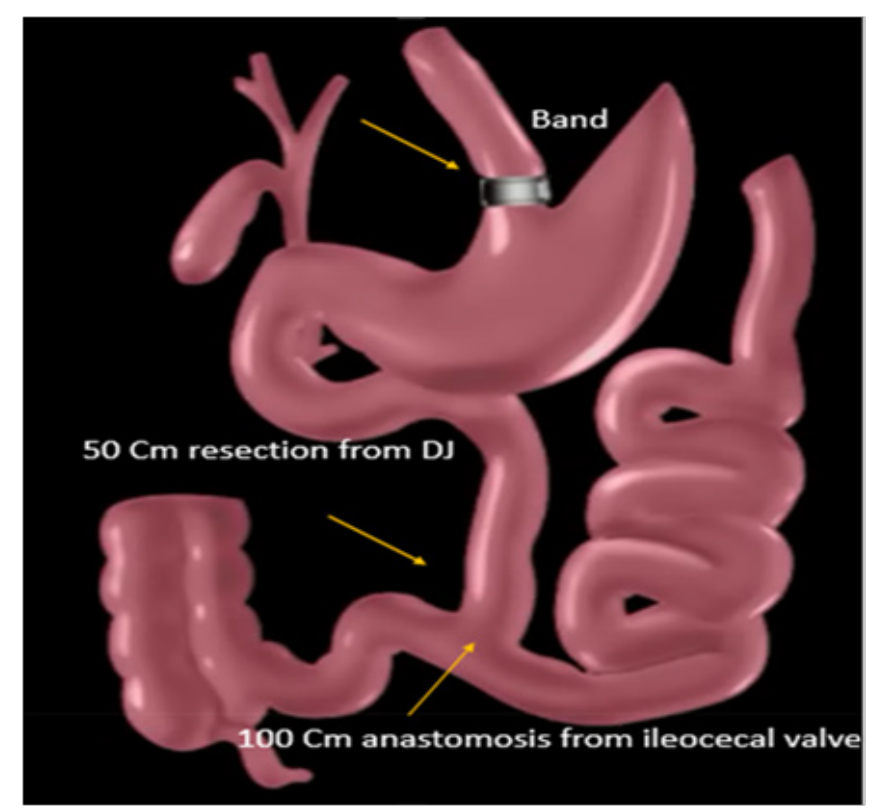

Figure 2 Elbanna operation, DJ, Dudeno-jejunal Junction.

The comorbidities of obesity in childhood include abnormalities in the endocrine, genital, cardiovascular, gastrointestinal, hepatic, pulmonary, orthopedic, neurologic, dermatologic, and psychosocial systems that affect both the quality of life and survival, should consider bariatric surgery if there is failure to control associated childhood -comorbidities, hence our preliminary results suggested bariatric surgery may be the clue in childhood morbid obesity, fact may drive a world to adopt guidelines for management of childhood related-comorbidities.

Considering the vertical transmission of microbiota may determine/ define early therapy, prevention therapy and alleviating bariatric procedures both in childhood and adulthood obesity, additionally surgical bypass- bariatrics would be considered in complicated childhood obesity, these items may determine childhood obesityrelated researches coming few years.

\section{Acknowledgements}

None.

\section{Conflict of interest}

The author declares no conflict of interest.

\section{References}

1. William J Klish. Definition; epidemiology; and etiology of obesity in children and adolescents. Up to date. 2016.

2. Christian Milani, Leonardo Mancabelli, Gabriele Andrea Lugli, et al. Exploring vertical transmission of bifidobacteria from mother to child. AEM. 2015;02037:15.

3. Ogden CL, Carroll MD, Kit BK, et al. Prevalence of childhood and adult obesity in the United States, 2011-2012. JAMA. 2014;311(8):806-814.

4. World Health Organization. Obesity: preventing and managing the global epidemic. Report of a WHO consultation. (World Health Organ Tech Rep Series (894), USA; 2000. 252 p.

5. Centers for Disease Control and Prevention (CDC). Behavioral Risk Factor Surveillance System Survey ACE Data. Atlanta, Georgia: US Department of Health and Human Services, Centers for Disease Control and Prevention. 2015.

6. Mechanick JI, Youdim A, Jones DB, et al. Clinical practice guidelines for the perioperative nutritional, metabolic, and nonsurgical support of the bariatric surgery patient-2013 update: cosponsored by American Association of Clinical Endocrinologists, the Obesity Society, and American Society for Metabolic \& Bariatric Surgery. Endocr Pract. 2013;19(2):337-372.

7. Peeters A, Barendregt JJ, Willekens F, et al. Obesity in adulthood and its consequences for life expectancy: a life-table analysis. Ann Intern Med. 2003;138(1):24-32.

8. Vlad I. Obesity costs UK economy 2 bn pounds sterling a year. $B M J$. 2003;327(7427):1308.

9. Duda RB, Darko R, Adanu RMK, et al. Prevalence of Obesity in an Urban West African Population: Results of the Women's Health Study of Accra. African J Health Sciences. 2007;14:154-159.

10. WHO. Obesity and overweight. 2015.

11. Freedman DS, Mei Z, Srinivasan SR, et al. Cardiovascular risk factors and excess adiposity among overweight children and adolescents:the Bogalusa Heart Study. J Pediatr. 2007;150(1):12-17.

12. Koren O, Goodrich JK, Cullender TC, et al. Host remodeling of the gut micro biome and metabolic changes during pregnancy. Cell. 2012;150(3):470-480.

13. Abd Elrazek Mohammad Ali, Abduh Elsayed Mohamed Elbanna, Shymaa E Bilasy. Medical management of patients after bariatric surgery: Principles and guidelines. World J Gastrointest Surg. 2014;6(11):220228

14. Must A, Jacques PF, Dallal GE, et al. Long-term morbidity and mortality of overweight adolescents. A follow-up of the Harvard Growth Study of 1922 to $1935 . N$ Engl J Med. 1992;327(19):1350-1355.

15. Skinner AC, Skelton JA. Prevalence and trends in obesity and severe obesity among children in the United States, 1999-2012. JAMA Pediatr. 2014;168(6):561-566.

16. Dinoto A, Marques TM, Sakamoto K, et al. Population dynamics of Bifidobacterium species in human feces during raffinose administration monitored by fluorescence in situ hybridization-flow cytometry. Appl Environ Microbiol. 2006;72(12):7739-7747. 
17. Elbanna A, Tawella N, Neff K, et al. Abstracts from the $18^{\text {th }}$ World Congress of the International Federation for the Surgery of Obesity \& Metabolic Disorders (IFSO), Istanbul, Turkey 28-31 August 2013. Obes Surg. 2013;23:1017-1243.

18. Elbanna A, Taweela NH, Gaber MB, et al. Medical Management of Patients with Modified Intestinal Bypass: A New Promising Procedure for Morbid Obesity. GJMR. 2014;14(1):8-19.
19. Abd Elrazek Ali Hussein, Abduh M Elbanna, Shymaa Bilasy, et al Can we avoid liver transplantation in NASH? A novel bypass surgery improves liver condition in NAFLD/NASH patients. AASLD. 2015.

20. Elbanna A1, Eldin MT, Fathy M, et al. Bariatric Bypass Surgery to Resolve Complicated Childhood Morbid Obesity: Case Report Study. Medicine (Baltimore). 2015;94(49):e2221. 